\title{
Research Development Using REDCap Software
}

\author{
Klauss Kleydmann Sabino Garcia ${ }^{1,2}$, Amanda Amaral Abrahão ${ }^{2}$ \\ ${ }^{1}$ Tropical Medicine Nucleus, Universidade de Brasilia, Brasilia, Brazil \\ ${ }^{2}$ Ministry of Health, Brasilia, Brazil
}

Objectives: High-quality clinical research is dependent on adequate design, methodology, and data collection. The utilization of electronic data capture (EDC) systems is recommended to optimize research data through proper management. This paper's objective is to present the procedures of REDCap (Research Electronic Data Capture), which supports research development, and to promote the utilization of this software among the scientific community. Methods: REDCap's web application version 10.4.1 released on 2021 (Vanderbilt University) is an EDC system suitable for clinical research development. This paper describes how to join the REDCap consortium and presents how to develop survey instruments and use them to collect and analyze data. Results: Since REDCap is a web application that stimulates knowledge-sharing among the scientific community, its development is not finished and it is constantly receiving updates to improve the system. REDCap's tools provide access control, audit trails, and data security to the research team. Conclusions: REDCap is a web application that can facilitate clinical research development, mainly in health fields, and reduce the costs of conducting research. Its tools allow researchers to make the best use of EDC components, such as data storage.

Keywords: Health Information Systems, Public Health Surveillance, Data Collection, Mobile Applications, Health Services Research

\section{Introduction}

High-quality clinical research is dependent on adequate design, methodology, and data collection [1]. A common part of clinical research and health surveillance is the dissemination of the information produced throughout the

Submitted: March 22, 2021

Revised: July 8, 2021

Accepted: August 11, 2021

\section{Corresponding Author}

Klauss Kleydmann Sabino Garcia

Tropical Medicine Nucleus, Universidade de Brasília, Campus Darcy Ribeiro, Asa Norte, Brasilia (DF) 70910-900, Brazil. Tel: +55 61 982193086, E-mail: kleydmann25@gmail.com (https://orcid. org/0000-0003-2268-8742)

This is an Open Access article distributed under the terms of the Creative Commons Attribution Non-Commercial License (http://creativecommons.org/licenses/by$\mathrm{nc} / 4.0 /$ ) which permits unrestricted non-commercial use, distribution, and reproduction in any medium, provided the original work is properly cited.

(c) 2021 The Korean Society of Medical Informatics process $[2,3]$. Electronic data capture methods and systems have been used in clinical research to improve data collection, storage, and analysis, and proper management tools are subsequently used for access control, audit trails, and data security [4-6].

The Research Electronic Data Capture (REDCap) platform is a web application that supports several types of clinical research, such as clinical trials, retrospective studies, and cohort studies, among others [7-13]. REDCap is compliant with FISMA (Federal Information Security Management Act), GDPR (General Data Protection Regulation), HIPAA (Health Insurance Portability and Accountability Act), and 21 CFR Part 11 (Part 11 of Title 21 of the Code of Federal Regulations). REDCap's consortium includes 5,200 partners spread across 141 countries (Figure 1), and it has been cited in over 14,500 articles (information updated in June 2021) [7-15].

This tutorial is motivated by the intention to disseminate a valid and detailed document for users ranging from begin- 


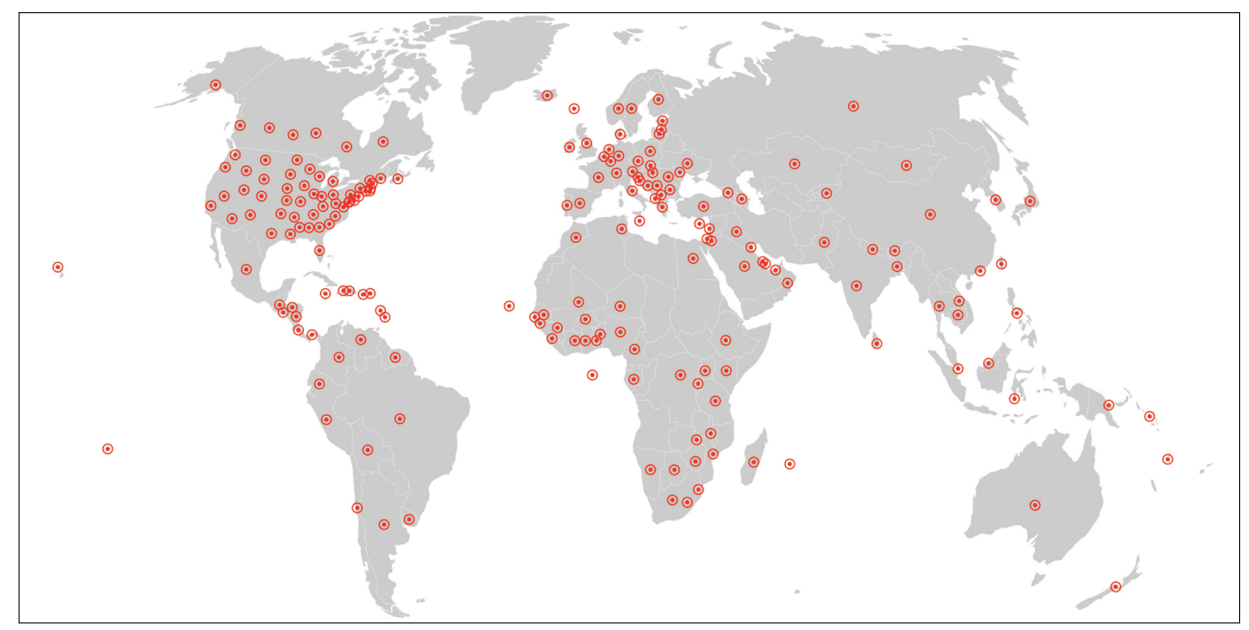

Figure 1. REDCap Consortium distribution across the globe.





Figure 2. REDCap user's new project configuration.

ners to highly-experienced scientists in clinical research. This article's objective is to present the major procedures involved in using REDCap to conduct research, as well as to promote utilization of the software among the scientific community.

\section{Methods}

\section{Joining the REDCap Consortium and Installation}

The REDCap software version described in this article is 10.4.1 released on 2021 (Vanderbilt University). REDCap software and consortium support are available at no charge to non-profit organizations that join the consortium. 
To obtain access to the REDCap software, the researcher must first contact the REDCap consortium to receive support and an adequate orientation through the following link: https://www.project-REDCap.org/partners/join/. To join, it is only necessary to agree to the standard license terms. If the researcher's institution already utilizes REDCap, the researcher must contact the institution's REDCap administrator to request the creation of a new user to $\log$ in to the system.

A REDCap requirement is to have a MYSQL client for performing the installation and updates. MySQL needs to be installed on a machine with a compatible operating system, such as Oracle Linux 8, Red Hat Enterprise 8, Debian 8, Ubuntu 21.04, or Microsoft Windows Server. A cloud solution for REDCap installation is an Amazon EC2 (Elastic Computing Cloud) instance with a compatible operating system. Cloud storage can be done through other Amazon Web Services (AWS) provided to avoid overloading the EC2 instance.

Institutions with limited infrastructure have the option of contacting the REDCap organization to have its administration carried out by a specific company at the cost of a

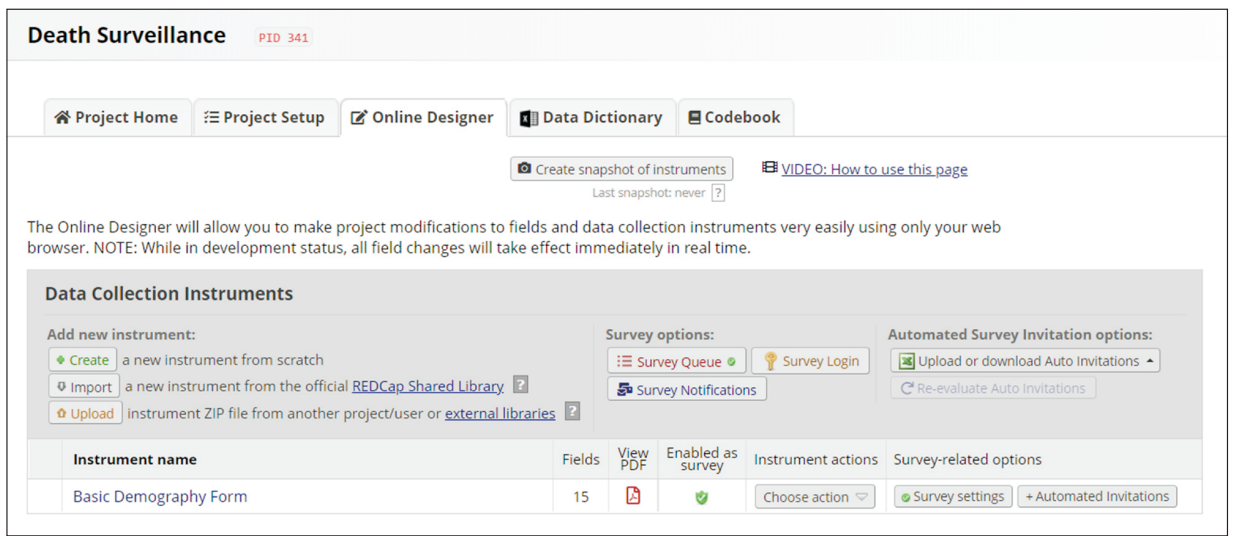

Death Surveillance PID 341

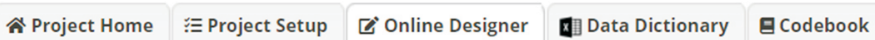

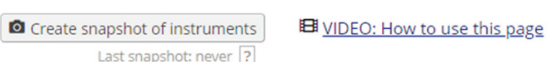

This page allows you to build and customize your data collection instruments one field at a time. You may add new fields or edit existing ones. New fields may be added by clicking the Add Field buttons. You can begin editing an existing field by clicking on the Edit icon. If you decide that you do not want to keep a field, you can simply delete it by clicking on the $\boldsymbol{X}$ Delete icon. To reorder the fields, simply drag and drop a field to a different position within the form below. NOTE: While in development status, all field changes will take effect immediately in real time.

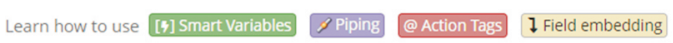

Current instrument: Basic Demography Form

Preview instrument

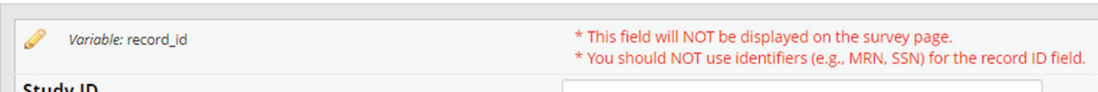

Study ID

* You should NOT use identifiers (e.g., MRN, SSN) for the record ID field.

NOTE: The field above is the record ID field and thus cannot be deleted or moved. It can only be edited

Add Field Add Matrix of Fields

$2 x$

Contact Information

Add Field Add Matrix of Fields

Q $\overrightarrow{4} \mathrm{X}$ Variable: first_name

First Name

Add Field Add Matrix of Fields

2 $\Rightarrow$ प 4 variable: last_name

Last Name

Add Field Add Matrix of Fields
Figure 3. Online designer menu.

Figure 4. Development of an instrument for data collection. 
monthly fee. Although a third-party company may administer the REDCap system, REDCap is not available for use by individuals without an institutional affiliation.
The opening page requires the researcher's login and password. After logging in, the user can start new projects.

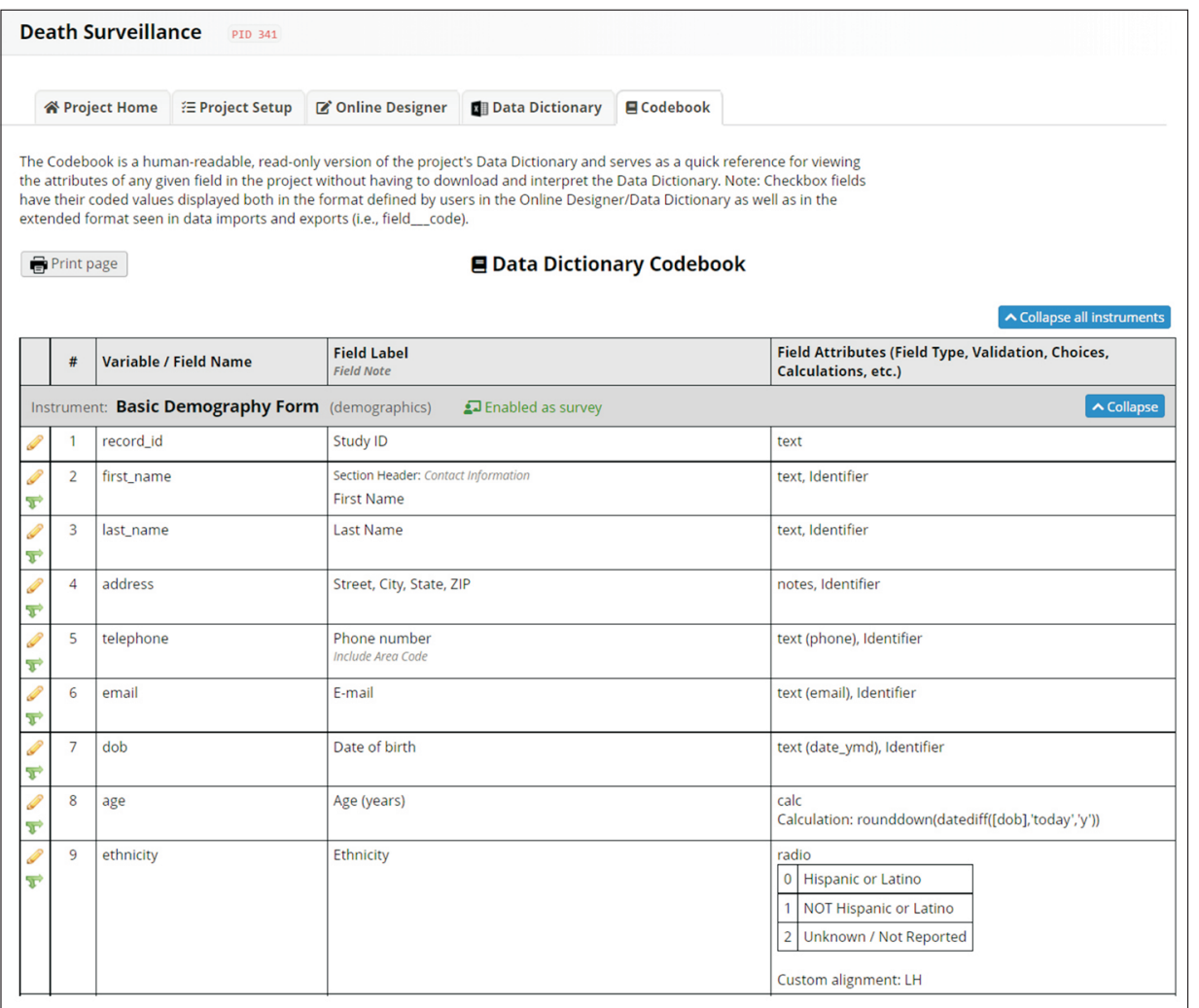

\section{Death Surveillance PID 341}

\section{Survey Distribution Tools}
SP Public Survey Link
a. Participant List
5. Survey Invitation Log

Using a public survey link is the simplest and fastest way to collect responses for your survey. You may obtain the survey link below to email it to your participants. Responses will be collected anonymously (unless the survey contains questions asking for identifying data from the participant). NOTE: Since this method uses a single survey link for all participants, it allows for the possibility of participants taking the survey multiple times, which may be necessary in some cases.

To obtain the survey link, copy the URL below and paste it into the body of an email message in your own email client. Your email recipient(s) can then click the link to begin taking your survey.

Public Survey URL: https://redcap.saude.gov.br/surveys/?S=NP7C9WHXR8

$\square$ Protect the public survey using the Google reCAPTCHA feature ?

\section{Link Actions}

\section{Open public survey}

Open public survey $+\hookrightarrow$ Log out

Send me URL via email

Survey Access Code or ㅁㅐㅁㅄㅇ QR Code
Figure 5. Instrument codebook and data dictionary.

Figure 6. Instrument distribution tools.

\section{Link Customizations}

Get Short Survey Link
Create Custom Survey Link
《i> Get Embed Code

Get Embed Code 


\section{Project Creation}

When the researcher first accesses REDCap, the initial page will present no projects to check. To initiate a project, the researcher must select "New Project" (Figure 2).

A new page will appear, on which the researcher must initialize the project configuration. A project title and project objective must be defined; examples of objectives would include "research" and "practice/just for fun." It is possible to insert notes about the project and to choose whether this new project will follow an existing template. REDCap has 15 pre-defined template options available with variables related to each theme (Figure 2). It is important to explore these templates before starting an official project to maximize their usefulness for the development of the study. However, it is advisable that if the project's questionnaires are already prepared, the research team should not use templates.

\section{Instrument Creation and Data Dictionary}

Once a project is ready to run, it is time to develop the data collection instrument. The "Online Designer" icon leads to the designer's menu (Figure 3). Then, one can select the "Create/a new instrument from scratch" option or edit an instrument that is already available if an initial template was selected in the previous steps. The user selects the instrument to be edited.

Next, it is possible to add questions through the "Add field" option (Figure 4). A new project page will open to allow field configuration. In this step, the user can choose question types such as "simple text," "calculated field," and "multiple choice," among others. Other configuration options, such as marking whether a field is required and whether it identifies participants, are required to finalize the question configuration.

Once an instrument's fields have been created, it is possible to reorganize them using drag-and-drop functionality. After the instrument is finalized, it is possible to check the "Codebook" (data dictionary) available in the tabs at the top of the page (Figure 5).

After checking whether the instrument is complete and accurate, the researcher must go to the "Project Setup" tab and click on the "Enable" icons in the "Main project setting" fields. The "I'm done!" icon must also be activated in the "Design your data collection instruments and enable your surveys" field. After the previous steps are concluded, a new

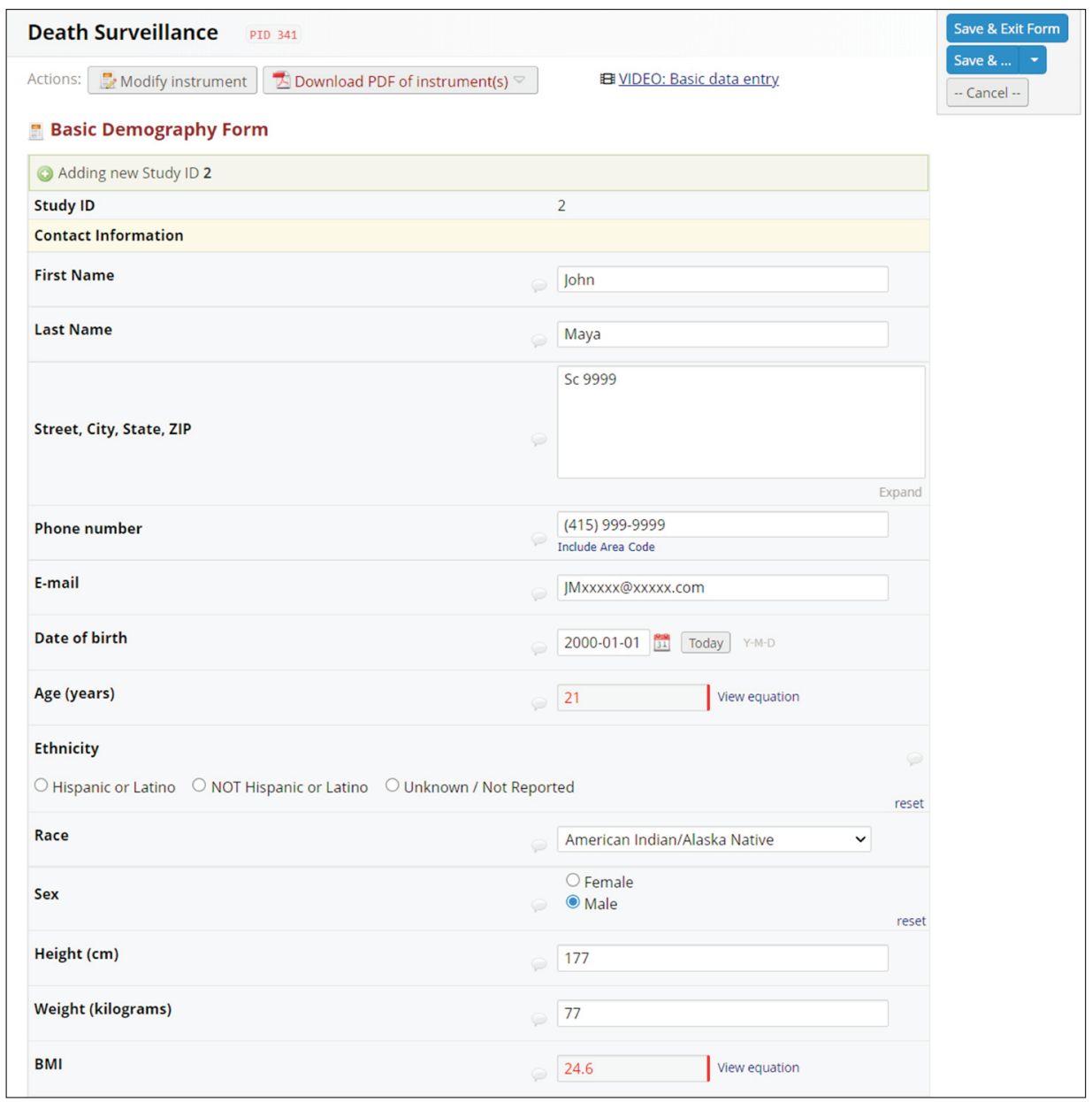


named field will be available in the side menu.

\section{Dissemination and Questionnaire Completion}

In the "Survey Distribution Tools" menu, the researcher can configure the distribution tools to disseminate the data collection instrument. The researcher must click on the "Enable" icon below the "Enabled as survey" column on the options for the generated instrument. A new window will be opened, where it will be possible to configure the survey title along with other tools such as "Survey Instructions" for the respondent. After the researcher presses "Save Changes," the software will return to the previous page where the icon "Survey Queue" must be selected to activate the instrument, and then dissemination to participants will be enabled. Once the previous step is completed, the researcher must select "Survey Distribution Tools" again. A new window will be opened where the researcher can copy the "Public Survey URL" or disseminate it through e-mail (Figure 6).



Figure 8. Data reports and exports menu.

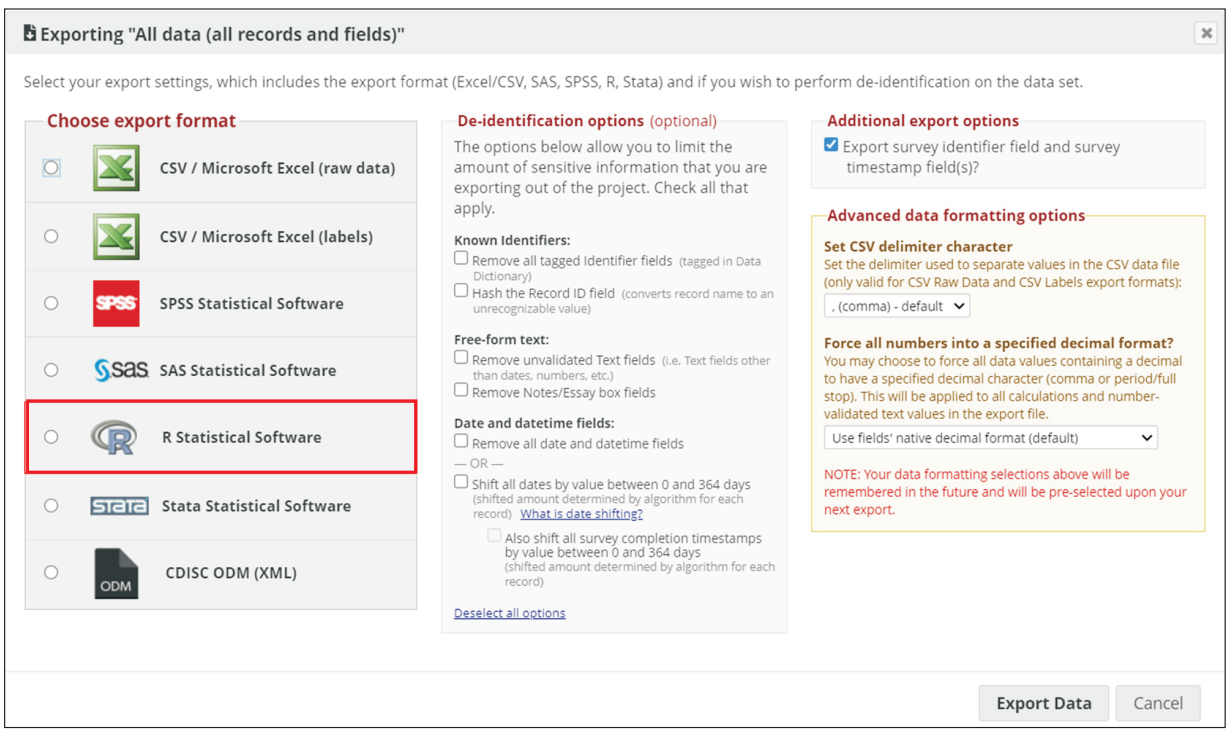

Figure 9. Data export configuration page. 


\section{Death Surveillance PID 341 \\ Â Project Home $\quad \vdots$ Project Setup User Rights ig: Data Access Groups}

This page may be used for granting users access to this project and for managing the user privileges of those users. You may also create roles to which you may assign users (optional). User roles are useful when you will have several users with the same privileges because they allow you to easily add many users to a role in a much faster manner than setting their user privileges individually. Roles are also a nice way to categorize users within a project. In the box below you may add/assign users or create new roles, and the table at the bottom allows you to make modifications to any existing user or role in the project, as well as view a glimpse of their user privileges.

Add new users: Give them custom user rights or assign them to a role.

\begin{tabular}{|c|c|c|}
\hline \multirow{2}{*}{$\Leftrightarrow$} & amanda.abrahao & Add with custom rights \\
\hline & $-O R-$ & \\
\hline & Assign new user & Assign to role \\
\hline
\end{tabular}

Create new roles: Add new user roles to which users may be assigned.

tos Enter new role name Create role

(e.g. Project Manager, Data Entry Person)

\begin{tabular}{|c|c|c|c|c|c|c|c|}
\hline $\begin{array}{l}\text { Role name } \\
\text { (click role name to edit } \\
\text { role) }\end{array}$ & $\begin{array}{l}\text { Username or users assigned to a role } \\
\text { (click username to edit or assien to role) }\end{array}$ & $\begin{array}{l}\text { Expiration } \\
\text { (click } \\
\text { expliation to } \\
\text { editt) }\end{array}$ & $\begin{array}{l}\text { Project } \\
\text { Design } \\
\text { and } \\
\text { Setup }\end{array}$ & $\begin{array}{l}\text { User } \\
\text { Rights }\end{array}$ & $\begin{array}{l}\text { Data } \\
\text { Access } \\
\text { Groups }\end{array}$ & $\begin{array}{l}\text { Data Export } \\
\text { Tool }\end{array}$ & $\begin{array}{l}\text { Reports \& } \\
\text { Report } \\
\text { Builder }\end{array}$ \\
\hline- & Admin.admin (Manager) & never & $\checkmark$ & $\checkmark$ & $\checkmark$ & Full Data Set & $\checkmark$ \\
\hline
\end{tabular}

You may set the rights for the user below by checking the boxes next to the application tools to which you wish to grant them access. You may also grant them or deny them access to individual data collection instruments, if so desired. To save your selections, click the "Add user" button at the bottom of the page.

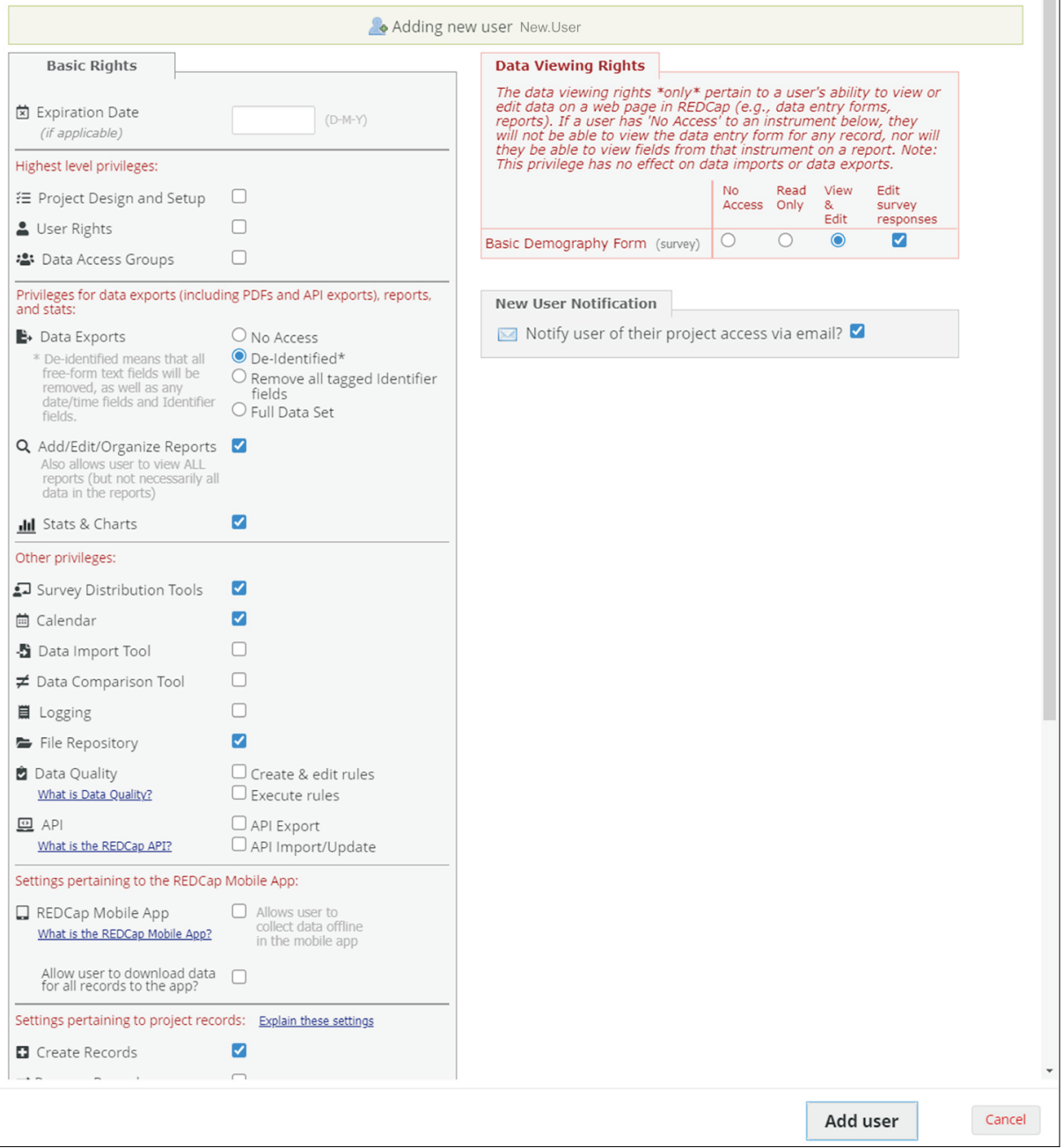

Figure 10. Addition of new users to the project and configuration of access levels. 


\section{Data Addition, Statistics, Exporting, and Reporting}

To input collected data manually, the researcher must access "Add/Edit Records" in the menu on the side. In the new window, the researcher must click on "Add new record." On the following page, the instrument will be available for data insertion. After completing all information, the instrument can be saved (Figure 7). In the "Add/Edit Records" menu, the answered questionnaires can be seen by selecting a record in the "Choose an Existing Study ID" option.

REDCap is available for desktop computers and mobile devices. The REDCap mobile application can be downloaded through a link on the side menu. The mobile app requires the creation of a new login user. Next, the instruments must be copied to the mobile device through the "REDCap Mobile App" option. The instruments can be transferred to the mobile device through a QR code. After data collection, the researcher must upload the data to the server using the mobile app functionality.

After running tests and before data collection, it is necessary to activate all project phases. If all steps have been completed and no other changes are required, it is time to activate "production mode." Activating "production mode" will block any project reconfiguration to prevent any unplanned modifications of the research. Only the system administrator can revert this process.

To check the data, the researcher must select "Data Exports, Reports, and Stats" in the side menu. From this menu, it is possible to view reports, export data, and check data statistics. The "View Report" icon shows all data that have been entered in a spreadsheet format containing all the questionnaire variables. By selecting the "Stats \& Charts" icon, a REDCap automatized visualization of questionnaire answers is shown as a descriptive analysis through tables and graphs (Figure 8). The "Export Data" option allows data extraction in comma-separated values format, as well as in specific statistical software formats for SPSS, SAS, Stata, and R [10] (Figure 9).

\section{Users' Permissions}

Through the "User Rights" option on the side menu, it is possible to configure access levels for projects and instruments. Using this menu, new users can be added (Figure 10). On this page, it is also possible to regulate permission levels, such as providing or denying access to some REDCap functionalities.

\section{Discussion}

The REDCap platform is intended to support clinical research [7]. Utilization of REDCap has grown substantially in epidemiology, especially in studies of coronavirus disease 2019 (COVID-19) [16-22]. The major advantages of using the REDCap software are long-term reduction of research costs, the possibility of utilization on both smart devices and desktop computers, and rapid data entry, review, and analysis [6].

REDCap's limitations are related to the need for a computer technician to perform maintenance, data backup, and application programming interface (API) configuration $[6,23]$. Despite the intuitive interface, the development of projects and instruments may be challenging for new users, making the learning curve slightly longer, and the access levels must be assigned carefully to ensure that no user deletes or changes data accidentally [7].

The utilization of REDCap improves data collection techniques, and REDCap provides a secure data storage system. REDCap has also been established as an appropriate tool for conducting clinical research and has been used widely across the globe by academic and governmental institutions [7-13].

\section{Conflict of Interest}

No potential conflict of interest relevant to this article was reported.

\section{ORCID}

Klauss Kleydmann Sabino Garcia (https://orcid.org/0000-0003-2268-8742) Amanda Amaral Abrahão (https://orcid.org/0000-0002-7537-7561)

\section{References}

1. Haug A, Zachariassen F, Van Liempd D. The costs of poor data quality. J Ind Eng Manag 2011;4(2):168-93.

2. World Health Organization. Report of the technical discussions at the twenty-first World Health Assembly on "national and global surveillance of communicable diseases". Geneva, Switzerland: World Health Organization; 1968.

3. Choi BC. The past, present, and future of public health surveillance. Scientifica (Cairo) 2012;2012:875253.

4. Backman C, Vanderloo S, Momtahan K, d'Entremont B, Freeman L, Kachuik L, et al. Implementation of an electronic data collection tool to monitor nursing-sensitive 
indicators in a large academic health sciences centre. Nurs Leadersh (Tor Ont) 2015;28(3):77-91.

5. Haux R. Health information systems: past, present, future. Int J Med Inform 2006;75(3-4):268-81.

6. Walther B, Hossin S, Townend J, Abernethy N, Parker D, Jeffries D. Comparison of electronic data capture (EDC) with the standard data capture method for clinical trial data. PLoS One 2011;6(9):e25348.

7. Patridge EF, Bardyn TP. Research electronic data capture (REDCap). J Med Libr Assoc 2018;106(1):142-4.

8. Oliveira SB, Ganem F, Araujo WN, Casabona J, Sanchez MN, Croda J. Imputation method to reduce undetected severe acute respiratory infection cases during the coronavirus disease outbreak in Brazil. Rev Soc Bras Med Trop 2020;53:e20200528.

9. Rufalco-Moutinho P, de Noronha LA, Quintao TD, Nobre TF, Cardoso AP, Ciliao-Alves DC, et al. Evidence of co-circulation of multiples arbovirus transmitted by Aedes Sp. based on laboratorial syndromic surveillance at health unit in slum area of federal district, Brazil [Internet]. Durham (NC): Research Square; 2021 [cited at 2021 Oct 6]. Available from: https://www.researchsquare.com/article/rs-588530/v1.

10. Duarte MM, Haslett MI, Freitas LJ, Gomes NT, Silva DC, Percio J, et al. Description of COVID-19 hospitalized health worker cases in the first nine weeks of the pandemic, Brazil, 2020. Epidemiol Serv Saude 2020; 29(5):e2020277.

11. Nishio EA, Cardoso ML, Salvador ME, D’Innocenzo M. Evaluation of Nursing Service Management Model applied in hospitals managed by social health organization. Rev Bras Enferm 2021;74(suppl 5):e20200876.

12. Leiria TL, Santos CB, Sant'anna RT, Trombetta JS, Osterkamp G, Kruse ML, et al. Analysis of conduction intervals in normal electrophysiological studies: establishment of reference values the Brazilian population. Int $J$ Cardiovasc Sci 2000;33:488-94.

13. Vaz J, Abelin AP, Schmidt MM, Oliveira PP, Gottschall CA, Rodrigues CG, et al. Creation and implementation of a prospective and multicentric database of patients with acute myocardial infarction: RIAM. Arq Bras Cardiol 2020;114(3):446-55.

14. Harris PA, Taylor R, Minor BL, Elliott V, Fernandez M, O’Neal L, et al. The REDCap consortium: building an international community of software platform partners. J Biomed Inform 2019;95:103208.

15. Harris PA, Taylor R, Thielke R, Payne J, Gonzalez N, Conde JG. Research electronic data capture (REDCap): a metadata-driven methodology and workflow process for providing translational research informatics support. J Biomed Inform 2009;42(2):377-81.

16. R Core Team. R: a language and environment for statistical computing [Internet]. Vienna, Austria: R Foundation for Statistical Computing; c2021 [cited 2021 Oct 5]. Available from: https://www.R-project.org/.

17. de Souza WM, Buss LF, Candido DD, Carrera JP, Li S, Zarebski AE, et al. Epidemiological and clinical characteristics of the COVID-19 epidemic in Brazil. Nat Hum Behav 2020;4(8):856-65.

18. Gadsden T, Bateman-Steel CR, Chaverot S, Ressler KA, Chee K, Redwood L, et al. Using a computerised database (REDCap) to monitor influenza vaccination coverage of healthcare workers and staff in South Eastern Sydney Local Health District. Aust Health Rev 2021; 45(1):97-103.

19. Maguire BJ, Guerin PJ. A living systematic review protocol for COVID-19 clinical trial registrations. Wellcome Open Res 2020;5:60.

20. Marcolino MS, Ziegelmann PK, Souza-Silva MV, Nascimento IJ, Oliveira LM, Monteiro LS, et al. Clinical characteristics and outcomes of patients hospitalized with COVID-19 in Brazil: results from the Brazilian COVID-19 registry. Int J Infect Dis 2021;107:300-10.

21. Koetter P, Pelton M, Gonzalo J, Du P, Exten C, Bogale $\mathrm{K}$, et al. Implementation and process of a COVID-19 contact tracing initiative: leveraging health professional students to extend the workforce during a pandemic. Am J Infect Control 2020;48(12):1451-6.

22. Gomides A, Ferreira G, Kakehasi A, Lacerda M, Marques C, Mota L, et al. Impact of chronic use of antimalarials on SARS-CoV-2 infection in patients with immune-mediated rheumatic diseases: protocol for a multicentric observational cohort study. JMIR Res Protoc 2020;9(10):e23532.

23. Crane S, Comer RS, Arenson AD, Draucker C. Using REDCap to facilitate web-based therapeutic intervention research. Nurs Res 2019;68(6):483-7. 